\title{
Mucosal melanomas in the elderly: challenging cases and review of the literature
}

\author{
This article was published in the following Dove Press journal: \\ Clinical Interventions in Aging \\ 12 June 2014 \\ Number of times this article has been viewed
}

\author{
Flavia Baderca ${ }^{1,2}$ \\ Dana Vincze ${ }^{3}$ \\ Nicolae Balica ${ }^{4}$ \\ Caius Solovan ${ }^{5,6}$ \\ 'Department of Microscopic \\ Morphology, Victor Babes University \\ of Medicine and Pharmacy, \\ Timisoara, Romania; ${ }^{2}$ Service of \\ Pathology, Emergency City Hospital, \\ Timisoara, Romania; ${ }^{3}$ Victor Babes \\ University of Medicine and Pharmacy, \\ Timisoara, Romania; ${ }^{4}$ Otolaryngology \\ Department, ${ }^{5}$ Department of \\ Dermatology, Victor Babes \\ University of Medicine and Pharmacy, \\ Timisoara, Romania; ${ }^{6}$ Department \\ of Dermatology, Emergency City \\ Hospital, Timisoara, Romania
}

\begin{abstract}
Melanomas are malignant tumors that originate from melanocytes. They are most frequently localized in the skin, but $5 \%$ of all melanomas interest also extracutaneous sites as mucosal surfaces, parenchymatous organs, the retroperitoneum area, and the ocular ball. The purpose of this study was to investigate the epidemiologic and morphologic data of mucosal melanomas diagnosed at Emergency City Hospital (Timisoara, Romania) during a period of 12 years. The study included 17 cases of extracutaneous, extraocular melanomas, with 16 primary melanomas and one secondary melanoma. All our patients were older than 53 years and were mostly men. Most of the patients presented with localized disease; only one case had regional lymph node metastases, and another one had systemic metastases at the time of diagnosis. Regarding localization, nine of 16 melanomas were in the head and neck region, six were diagnosed in the gastrointestinal and urogenital tracts (three cases each), and one case had a rare localization (retroperitoneum). The most common histologic type was represented by epithelioid cells, and the majority of the tumors were achromic. Mucosal melanoma is a tumor associated with aging, all our patients being older than 53 years. Because of unspecific symptoms and low incidence, the diagnosis is often delayed and requires teamwork among the clinician, pathologist, radiologist, and oncologist. Different genetic fingerprints impose a correct diagnosis to offer the patient the best novel, personalized therapy.
\end{abstract}

Keywords: mucosal melanoma, melanocytes, molecular classification, kit gene mutations, immunohistochemistry

\section{Introduction}

Melanomas can arise anywhere in the body, originating from melanin-producing cells called melanocytes. Even though the most common localization is the skin, 5\% of all melanomas appear in other sites of the body, including the ocular ball and, in fact, all mucosal surfaces. A very small percentage of melanomas can be diagnosed in parenchymatous organs, such as the lung, or in the retroperitoneal area. ${ }^{1}$

The first mucosal melanoma was cited in 1859 by Weber and was later recognized as a distinct entity in 1869 by Lucke. Lincoln, in 1885, recorded the first mucosal melanoma in the English literature. Because of its rarity, this entity is poorly recognized, described, and understood..$^{2-4}$

Cutaneous and noncutaneous melanomas share the same origin cells but have divergent etiology, behavior, and molecular biology. Nowadays, genetic studies have demonstrated that melanomas are not a disease but are a family of diseases, with mucosal melanomas playing a distinct, important, and specific role. ${ }^{5}$

\section{The aim of the study}

The purpose of the study was to investigate the epidemiologic and morphologic data of mucosal melanomas diagnosed at Emergency City Hospital (Timisoara, Romania) during a period of 12 years.
Correspondence: Flavia Baderca

52 Cozia Street, 300580, Timisoara,

Romania

$\mathrm{Tel}+40$ 733I 06I0 I

$\mathrm{Fax}+40256204900$

Email flaviabaderca@yahoo.com 


\section{Materials and methods}

We retrospectively analyzed 186,111 patient files recorded in our pathology service during a period of 10 years, from January 2002 to December 2011, identifying 15 cases of mucosal melanomas. The slides were searched from the archive and reread by two individual pathologists. While preparing the study design, we also reviewed 35,815 histological slides read between January 2012 and June 2013 and selected two cases of possible mucosal melanomas.

The final group included 17 cases of mucosal melanoma admitted in different departments of Timisoara's Emergency City Hospital from 2002 to 2013. After inspection of the recorded files, the pathologists noted the localization of the tumor, symptoms if available, and gross anatomy features. At the time of diagnosis, all cases were thoroughly searched to determine the cutaneous origin of the melanoma. For diagnostic purposes, all the biopsied tissue specimens were initially processed with routine histological technique, with the archive slides being stained with hematoxylin and eosin. While reading the slides, the pathologists paid attention to the histological type of the cells, cellular and nuclear pleomorphism, mitotic count, and quantity of melanin pigment. If they considered it necessary, for achromic tumors, additional immunohistochemical stains for different markers (melanocytes [HMB45, Melan A, S100], epithelia [AE1/ AE3], neuroendocrine cells [chromogranin], mesenchymal origin [CD34] and cell proliferation [Ki67]) were performed, using the protocol of the antibody producer Novocastra Reagents and Antibodies: Leica Biosystems, New Castle, UK.

\section{Results}

In the period studied, we identified 16 primary mucosal melanomas and one secondary pleural melanoma of unknown origin. All the data regarding clinical, epidemiological, and histopathological aspects of the cases are summarized in Table 1. Eleven of our patients were smokers.

For the primary mucosal melanomas, the male/female ratio was 10:6. At the time of diagnosis, the age for men ranged between 53 and 94 years (median, 73.5 years), and for women, age ranged between 53 and 73 years (median, 63 years). Regarding localization, nine of 16 melanomas were from the head and neck region, six were diagnosed in the gastrointestinal and urogenital tracts (three cases each), and one case had a rare localization (retroperitoneum). From these cases, one case from the urogenital melanoma group presented with a pelvic tumor that included ovary and uterus, making it impossible to determine the organ of origin.
Between head and neck melanomas, we diagnosed one case of laryngeal mucosa melanoma, with the other cases being divided equally between the nasal cavity and oral mucosa (four cases each), with a clear predominance of male patients (seven of nine patients). The most frequent symptoms encountered were tumor presence and bleeding because of ulceration. The dominating cell type was epithelioid (seven cases); in only two cases of head and neck mucosal melanomas did we observe both types of cells (epithelioid and spindle). In this group, we diagnosed six cases of achromic melanomas; the other three were pigmented, with areas composed of heavily pigmented cells admixed with achromic zones. The epithelioid cells were intensely pleomorphic, whereas the spindle cells had a monomorphic appearance. The mitotic index was high (18 mitoses/10 high power fields) for the epithelioid cells tumors, with many atypical mitotic figures. At diagnosis, seven cases had localized tumor without any evidence of lymph node metastases, one showed regional lymph node metastases, and another showed concomitant systemic metastases.

From this group, we decided to present four cases we considered more challenging. In case 9, a 94-year-old man was diagnosed with lower lip mucosal melanoma. This was the oldest patient of our group, and at the time of diagnosis, he presented with metastases in three regional lymph nodes. Moreover, 6 months after the diagnosis, he returned with submandibular lymph node metastasis consistent with a pigmented melanoma with epithelioid cells (Figure 1).

In case 7, at presentation, a 53-year-old man complained about right palatine tonsil bleeding, odynophagia, jaundice, and melena. The laboratory tests revealed anemia. An otolaryngology clinic exam suspected a T2 tumor, approximately $3 \mathrm{~cm}$ in largest diameter (Figure 2), and an excision biopsy (right tonsillectomy) was performed. Histopathologic exam revealed a malignant tumor composed of epithelioid cells with one or more high pleomorphic nuclei and large nucleoli (Figure 3). Some cells presented eosinophilic macronucleoli. No pigment was identified in the cytoplasm of malignant cells. At that time, the diagnosis was undifferentiated carcinoma.

The patient presented with postoperative melena, jaundice, and low hemoglobin and hematocrit blood levels, despite the lack of oropharyngeal bleeding. A general surgery exam was requested, and gastroscopy showed a gastric tumor. The patient was admitted into the general surgery department, where the gastric tumor was excised and the microscopic inspection of the surgical specimen highlighted multiple pigmented mucosal metastases at the gastric and ileal levels. The case was reevaluated and reconsidered as 


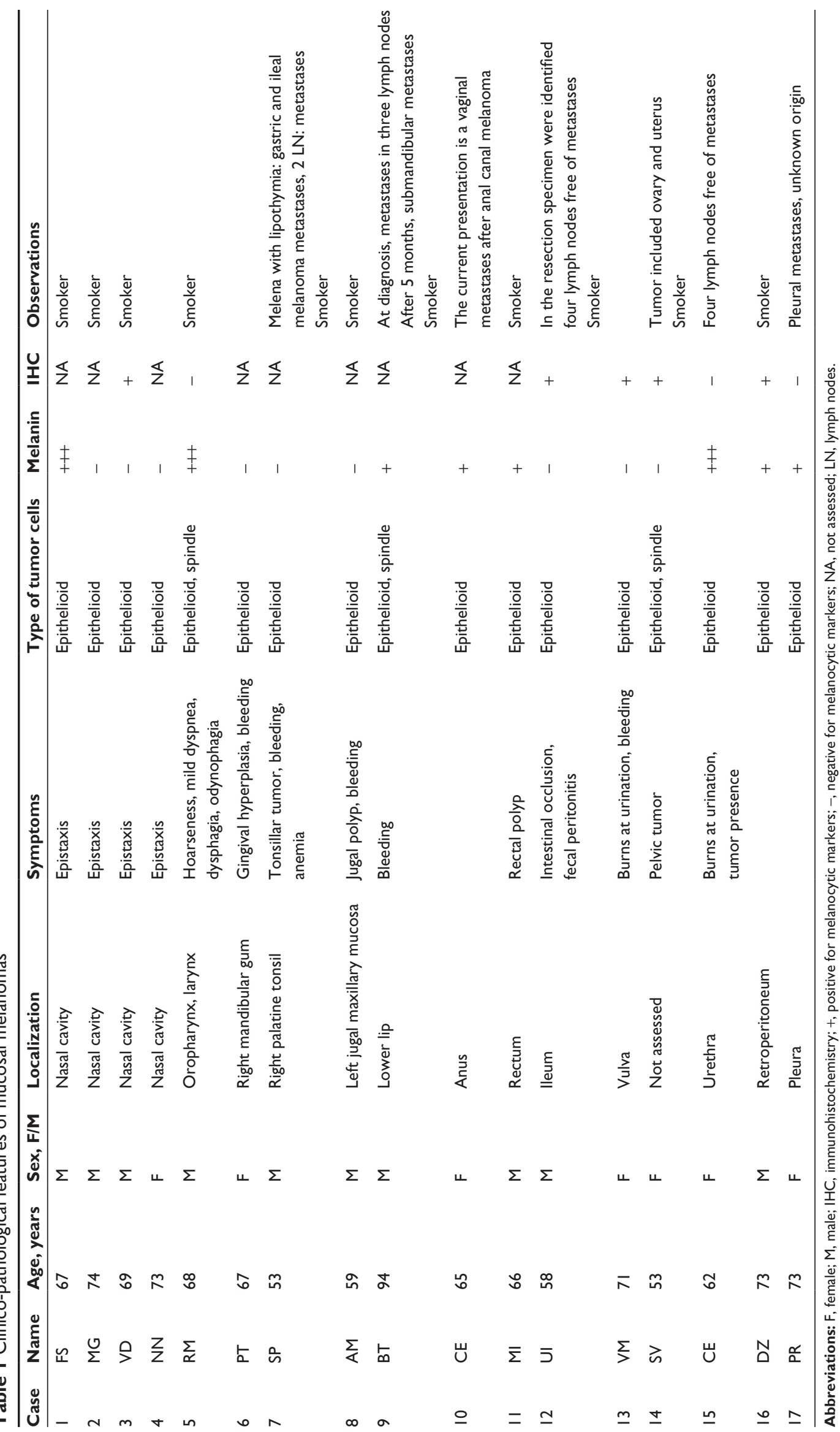




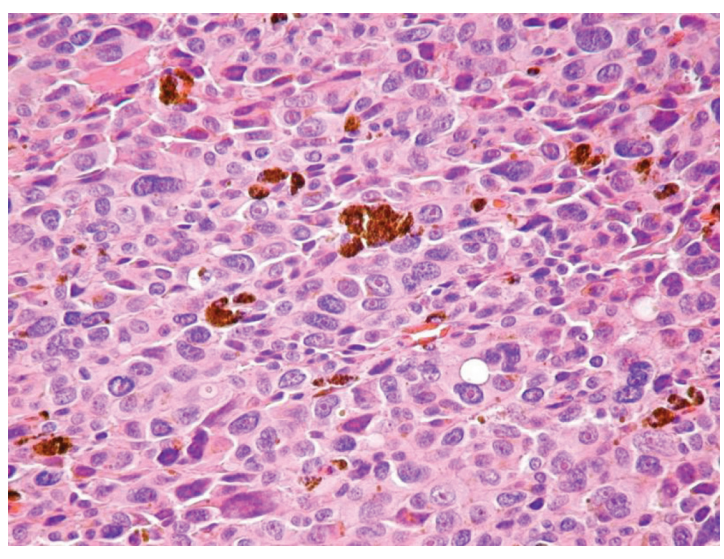

Figure I Pigmented melanoma metastasis constituted from pleomorphic epithelioid cells; hematoxylin and eosin stain, ob. 40x.

Abbreviation: ob, objective.

a primary palatine tonsillar achromic melanoma with concomitant pigmented gastric and ileal metastases.

The first case was of a 67-year-old man who presented to the otolaryngology department for recurrent epistaxis from left nasal fossa, left nasal obstruction and crusts, left ear fullness, and low-frequency left ear tinnitus. Otolaryngology clinical exam revealed a left nasal fossa polypoid mass arising from the level of middle meatus, extending to the choana (Figure 4). An enhanced computed tomography scan with coronal reconstruction was performed, revealing the tumor's extension into the left nasal fossa (lateral wall) without involvement of the paranasal sinuses. A tumor resection was performed by paralateronasal incision with wide margins. The microscopic appearance was consistent with heavy pigmented mucosal melanoma with epithelioid cells, which was a peculiarity of the case, as all three other cases of nasal cavity melanomas were achromic tumors.

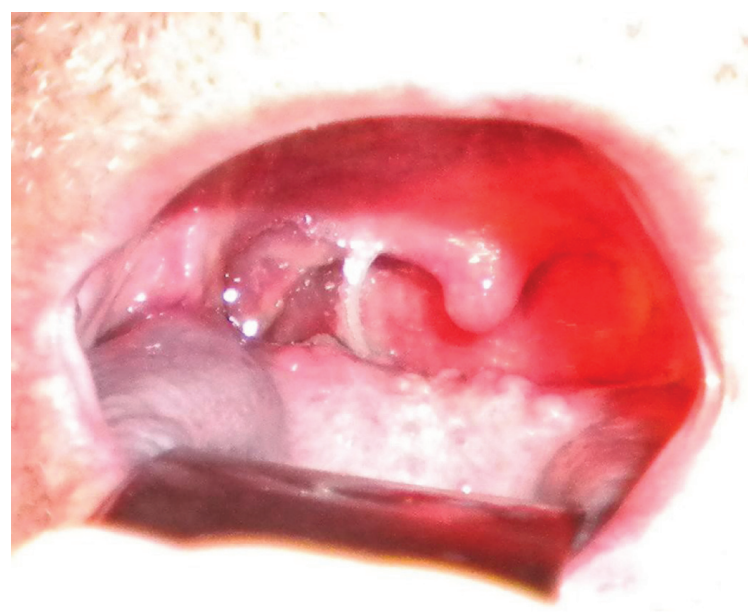

Figure 2 Bucopharyngoscopy: oropharyngeal (right palatine tonsil) melanoma.

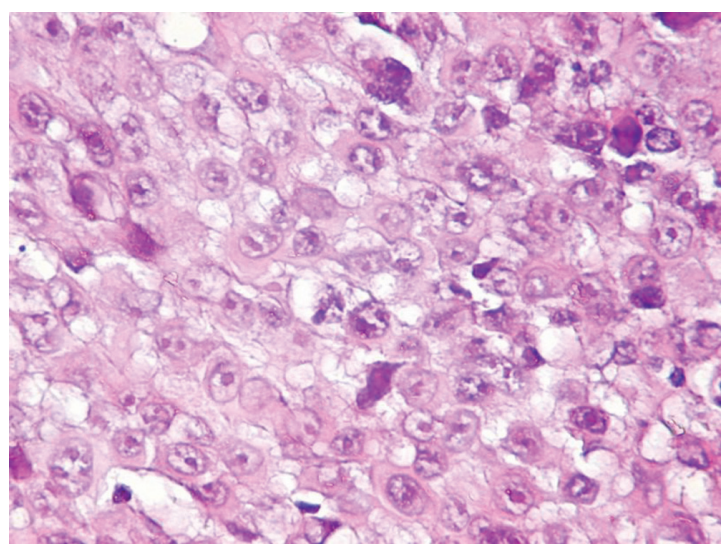

Figure 3 Epithelioid malignant melanocytes with high pleomorphic nuclei and large macronucleoli; hematoxylin and eosin stain, ob. 40x.

Abbreviation: ob, objective.

In case 5, the 68-year-old, male patient presented with hoarseness, mild dyspnea, dysphagia, and odynophagia that had been in place for approximately 3 months. Otolaryngology clinic exam by indirect laryngoscopy and $70^{\circ}$ rigid pharyngolaryngoscopy (Figure 5) revealed an infiltrative vegetative tumor that involved the laryngeal vestibule and the hypopharyngeal retrocricoid area, with normal vocal cord mobility and no cervical lymph node involvement. A biopsy was performed by suspended microlaryngoscopy, and the microscopic investigations showed a malignant tumor with characteristics consistent with heavy pigmented primary mucosal melanoma of the larynx, composed of a mixture of epithelioid and spindle cells.

In the gastrointestinal mucosal melanoma group, all patients identified had localized disease, but a 65-yearold woman returned 12 months from the diagnosis with vaginal metastases. At microscopic level, all the tumors had

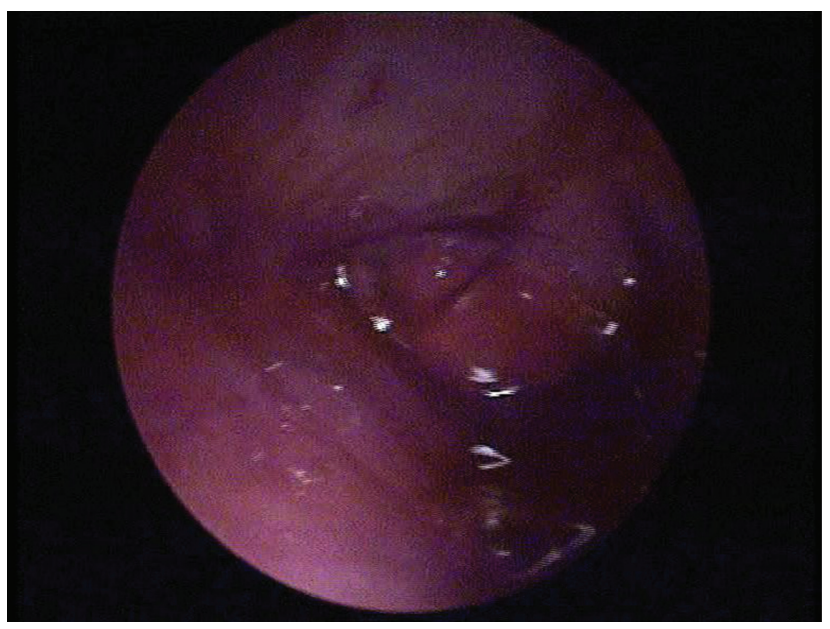

Figure 4 Left nasal fossa polypoid mass. 


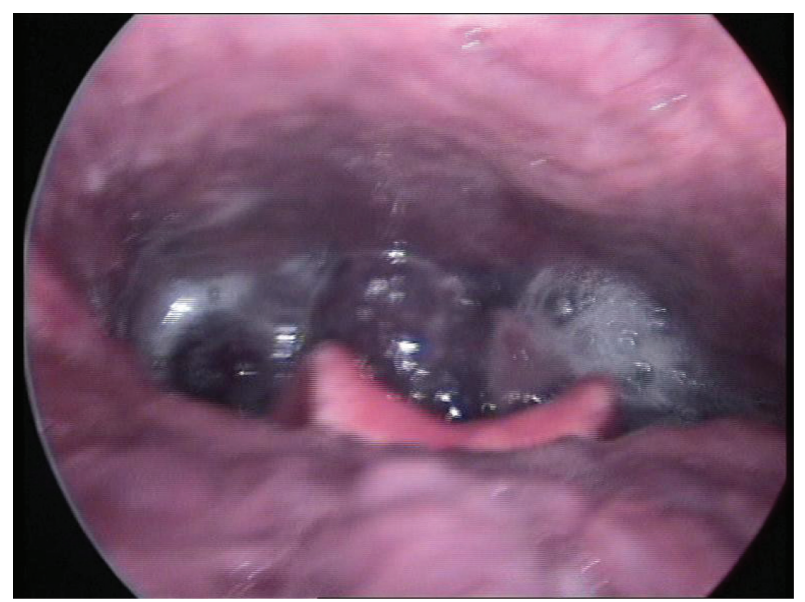

Figure $570^{\circ}$ rigid hypopharyngolaryngoscopy: laryngeal melanoma.

epithelioid cells, two contained small quantities of melanin in the cytoplasm of malignant melanocytes, and one was an achromic tumor that required further immunohistochemical tests for diagnosis.

In the urogenital mucosal melanoma group, all the patients were women. Two presented at diagnosis with localized disease, and a 53-year-old woman presented with a large tumor that included the left ovary and the uterus. The microscopic investigation of the biopsied specimens revealed a malignant tumor composed of epithelioid and spindle cells with no evidence of pigment deposits. Further immunohistochemical reactions positive for melanocytic markers established the correct diagnosis (Figure 6).

We diagnosed a 73-year-old man with a pigmented retroperitoneal melanoma, a rare localization for a primary extracutaneous melanoma. The cells contained a small quantity of pigment and were immunohistochemically positive for melanocytic markers.

\section{Discussion Epidemiology}

Mucosal melanoma is a rare disease, but its incidence seems to remain stable. ${ }^{6-8}$

Most authors appreciate that there are some racial and regional differences in the incidence of mucosal melanomas. In other countries, mucosal melanomas affect black patients with a higher prevalence, at $11.8 \%$ versus $1.3 \%$ in whites. ${ }^{8}$ In our group, all the patients were white because of geographic regional considerations.

Regarding the incidence of mucosal melanomas in specific parts of the body, it has been shown that the lesions of oral cavity are more frequent in Japanese, where they account for $35 \%$ of all mucosal melanomas, which is much

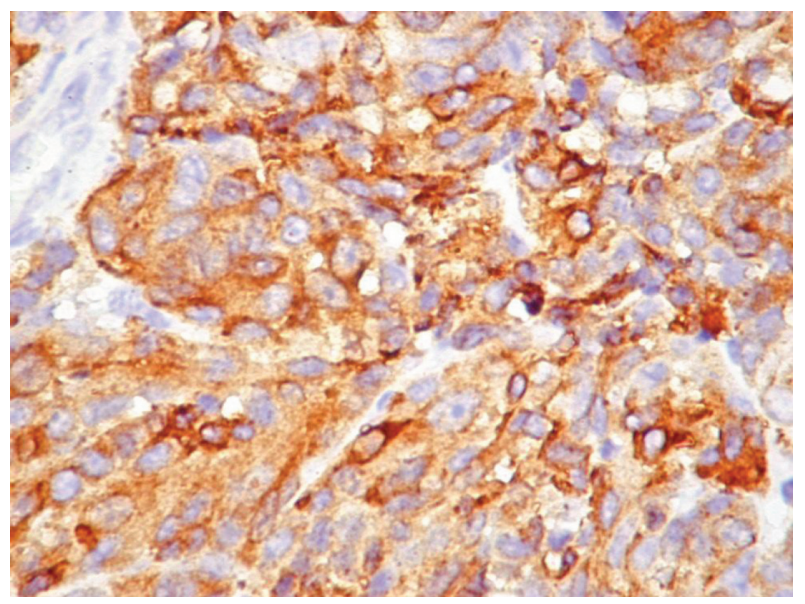

Figure 6 Melan A positivity in the malignant melanocytes, ob. 40x. Abbreviation: ob, objective.

more that the incidence of mucosal melanomas in whites, at $3.6 \%$, reported worldwide. ${ }^{8-11}$ In addition, another study reported a higher incidence of oral cavity and sinonasal melanomas in Uganda. ${ }^{12}$ In our study, we found a relatively high incidence of oral cavity melanomas (25\%) compared with that seen in the literature.

McLaughlin et $\mathrm{al}^{6}$ showed that in US, mucosal melanomas appear more frequently in women than in men. They assumed this higher incidence in women indicated a preferential appearance of urogenital melanomas in female patients. All our cases of urogenital melanomas were in women, although in the studied group, there was a contrary sex predilection compared with data from the literature, with 10 of 16 patients being men.

Mucosal melanomas are lesions associated with aging, appearing at older ages compared with their cutaneous counterparts. Some authors report that more than $60 \%$ of patients are older than 60 years, with only $3 \%$ being younger than 30 years. $^{6}$ At diagnosis, 12 of 16 patients were older than 60 years. We did not diagnose any case of mucosal melanoma in persons younger than 50 years.

\section{The cell of origin}

The tumors originate from melanocytes, which are cells derived from the neural crest. During gastrulation, melanocyte precursors migrate through mesenchymal tissue to their final destination. In addition to cutaneous localization, on mucosal surfaces of the human body, melanocytes are more frequently observed in tissues originating from the neuroectoderm, such as the nasopharynx, larynx, trachea, and bronchia, than from nonectodermal tissues, such as the gastrointestinal and urogenital tracts. Even if cutaneous melanomas are more common than mucosal melanomas, the 
number of melanocytes on $1 \mathrm{~mm}^{2}$ in the skin of the abdomen is half that of oral or nasal cavity mucosa.

\section{Etiologic factors}

Until now, the etiologic factors of mucosal melanomas have not been identified. Sun exposure, which is a well-known risk factor for cutaneous melanomas, seems to play no role in the pathogenesis of nonskin melanomas. Genetic studies have shown that other irritants and carcinogenic factors have no fingerprint on the etiology of noncutaneous melanomas. There are some exceptions, however. For example, tobacco smoke is considered to be implicated in the pathogenesis of oral melanomas. ${ }^{10,13-18}$ Moreover, it was already shown that cigarette smoking determinates the melanocytes' hyperplasia of oral mucosa, with one third of melanomas arising in this location being preceded by oral melanosis for 10 or 20 years. $^{10,13-18}$ The diagnosis and careful observation of mucosal melanosis is essential for an early detection of its malignant transformation, with lots of scientists emphasizing that this entity preceded, for many years, the onset of mucosal melanoma, consisting of a precursor, premalignant lesion. The same behavior was observed in ocular melanocytic lesions. ${ }^{10,13-18}$ Another irritant found to have an important role in the etiology of mucosal melanoma is formaldehyde, with professional exposure to its vapors being implicated in the genesis of sinonasal melanomas. ${ }^{19,20}$

\section{Cytogenetics}

It is well-known that a $B R A F$ mutation is involved in the pathogenesis of $75 \%$ of skin melanomas, ${ }^{21-23}$ but this status is identified in only a small percentage of mucosal melanomas. ${ }^{5}$ Instead of this, c-kit mutations have been noticed, by many authors, in $40 \%$ of mucosal melanomas, with the pioneer in this field being Professor Richard Marais, director of the Paterson Institute for Cancer Research and lead author of the DNA sequencing research carried out at Cancer Research UK's Paterson Institute for Cancer Research at the University of Manchester who recently concluded, using genome sequencing, the distinct mechanism that drives mucosal melanomas and clearly differentiates them from cutaneous counterpart. ${ }^{24-28}$ Some authors also noticed different c-kit overexpression between mucosal melanomas in various sites. c-kit expression has been found in $88 \%$ of oral mucosa melanoma, whereas only $12 \%$ of anal mucosa melanomas harbor this feature. ${ }^{26,27}$ These findings, together with the discovery of distinct GNAQ or GNA11 implication in $80 \%$ of uveal melanomas, support the idea of the importance of the molecular classification of melanomas. , $29,30^{2}$

\section{Sites of involvement}

Half of mucosal melanomas appear in the head and neck region, with the rest being shared equally between the gastrointestinal and urogenital tract locations. In head and neck melanomas, the most common are those arising from upper respiratory tract $(55 \%)$, followed by the oral cavity (40\%), and a small percentage of cases from the pharynx and larynx. ${ }^{15,31-34}$ The high prevalence of head and neck melanomas in our study (nine of 16 cases) resulted from the high incidence of oral cavity melanomas (four cases). We consider this incidence to be supported by habitual characteristics; eg, in our country, cigarette smoking unfortunately is still very widespread. The literature notes that among upper respiratory tract melanomas, about $80 \%$ occur in nasal cavity, whereas only $20 \%$ show sinuses mucosa as the site of origin. Through oral cavity structures, the hard palate and maxillary alveolus are most affected. ${ }^{31}$ One-third of oral melanomas develop from preexisting melanocytic lesions. ${ }^{33}$

The second most frequent place is the gastrointestinal tract, in which melanomas were reported with an incidence of $25 \%$ from all mucosal melanomas, with the decreasing order of appearance being the anorectal mucosa, the esophagus, and the small bowel. Only a few gastric cases were published in the English literature, despite the fact that the first case of mucosal melanoma described in 1885 by Lincoln had gastric localization. ${ }^{45-42}$ An interesting particularity of our study was represented by a case of palatine tonsil melanoma in a 53-year-old man, the youngest of our patients, which was at presentation classified in the head and neck group of patients. After surgical removal of a tonsillar tumor, the symptoms did not cease (melena, jaundice), and he was readmitted in our hospital. Further investigation revealed multiple gastric and ileal metastases. Moreover, despite the primary tumor being achromic, which is somehow characteristic for mucosal melanomas, the further mucosal metastases were heavily pigmented.

Melanomas of the urogenital tract have almost the same incidence as those arising from the gastrointestinal tract, representing $20 \%$ of all mucosal melanomas, but are more common among women. ${ }^{43}$ In fact, our three cases of urogenital-located melanomas were all in women. We diagnosed two primary mucosal melanomas: one in the glabrous skin of labia majora and one with urethral localization. The third case was a secondary tumor with vaginal localization after a primary anal mucosa melanoma in a 65-year-old woman. We did not find another case of mucosal metastases after mucosal melanoma in the English literature. It was stated that vulvar localization is the most common location, 
followed by the vagina, uterine cervix, urethra, and urinary bladder. ${ }^{6,7}$ In our study, the urethral melanoma was composed of heavily pigmented malignant cells, an aspect that differed from what we found in most mucosal melanomas. This aspect obliged us to categorize the case as an animaltype melanoma. ${ }^{44}$

Very few cases of extracutaneous melanomas of parenchymatous organs were published in the literature. The melanomas of retroperitoneal area and parenchymatous organs such as lung or prostate cover $5 \%$ of all malignant melanocytic tumors, with extracutaneous localization. ${ }^{7}$ In our study, we included two cases with rare localization (one retroperitoneal, considered a primary melanoma, and another pleural, diagnosed as a metastatic melanoma). Knowing that extracutaneous and cutaneous melanomas have distinct genetic fingerprints, we take into consideration further studies of comparative genomic hybridization to establish with better probability the extracutaneous vs cutaneous origin.

The symptoms of mucosal melanomas are unspecific. Almost always the clinician dealing with a patient who presented with a lump or a polyp does not have in mind a mucosal melanoma as the first suspected diagnosis.

In the head and neck region, for nasal cavity melanomas, the most common symptoms are obstructive, because of the presence of a bleeding unilateral mass. In most cases, the gross inspection of the tumor reveals an achromic lesion. The oral cavity melanomas can be pigmented or not. In early stages, the tumors are flat, and elevation, ulceration, bleeding, and teeth loss appear after a long period of evolution. For $30 \%$ of oral cavity melanomas, the physicians can demonstrate the presence of a preexisting melanocytic lesion that preceded the tumor for many years. The careful inspection of all pigmented lesions of oral mucosa is mandatory to diagnose a malignant transformation from the beginning or in early stages.

For gastrointestinal tract melanomas, the symptoms are also unspecific. In the anorectal region, the mucosal melanomas almost always appear as a polypoid mass that is responsible for rectal bleeding, discomfort at defecation, and abdominal pain. Primary intestinal and gastric melanomas are very rare, and physicians should always carefully search for the existence of a malignant cutaneous melanocytic tumor to differentiate metastasis from primary tumor. A gastric ulcer that does not heal for many years under appropriate treatment, or with an unusual presentation, should gain the attention of the clinicians to correctly diagnose a mucosal melanoma. Other symptoms described in the literature are weight loss and secondary anemia. ${ }^{7}$
Small bowel is the election site of cutaneous melanomas metastasis, so the presence of a melanocytic tumor at this level should alert gastroenterologists to search for a history of skin melanoma, or even a concurrent cutaneous lesion. ${ }^{45-47}$ The cited symptoms in the literature are abdominal pain, anorexia, nausea, vomiting, and intestinal bleeding with secondary anemia. ${ }^{7,48}$ In our study, we identify two cases of melanomas of the ileum. One was primary, in a 58-year-old man, and the other was a metastasis of a palatine tonsillar melanoma in a 53-year-old man.

In urogenital melanomas, vulvar melanomas are the most frequent. They share the same symptoms with other malignancies at this level, so pruritus, irritation, vaginal discharge, and discomfort when urinating, together with the presence of a mass at clinical inspection that is sometimes ulcerated or bleeding and frequently not pigmented. The signs are not specific indicators of primary vulvar melanomas, with carcinoma usually being the first diagnosis in the clinician's mind. ${ }^{49-51}$ Our case of vulvar melanoma was represented by a 71-year-old woman. On clinical grounds, the first diagnosis suspected was undifferentiated carcinoma. Histopathological examination revealed that the tumor was composed of sheets of moderately pigmented malignant melanocytes; therefore, the diagnosis of melanoma was imposed. The existence of intraepithelial melanocytic precursor lesions led to the diagnosis to primary mucosal melanoma. Primary mucosal melanomas of the urethra and urinary bladder are extremely rare. These tumors most frequently appear in women and usually are polypoid and achromic, sometimes asymptomatic and sometimes showing hematuria, dysuria, weight loss, and secondary anemia. ${ }^{52-55}$ The first diagnosis suspected is a urethral polyp or urothelial carcinoma. In our study, we identified one case of urethral melanoma that was clinically considered a vaginal polyp. Its correct location was proven during surgery, when the clinician observed a long stalk that implanted the tumor in the urethral mucosa. Extremely rare for mucosal melanoma, this case was histologically composed of heavily pigmented melanocytes somehow similar to the aspect observed in the ocular melanomas, in blue nevi (either benign or malignant), and in animal-type melanomas. ${ }^{56,57}$

Because its symptoms are unspecific and its localization in the majority of cases is hidden, the diagnosis of mucosal melanoma is always tardive and represents a real challenge for physicians. Moreover, it requires teamwork among clinicians, radiologists, and pathologists. Stubbornly biopsying all unusual tumors with regard to clinical presentation, behavior, and unresponsiveness to the treatment, is the key 
for early detection of mucosal melanomas, knowing that this disease has a far more aggressive course than its cutaneous counterpart. The careful examination by the pathologist of the excised specimens and the use of immunohistochemical stains are mandatory to complete diagnosis and open new doors for modern treatment methods.

For a long time, the mainstay of treatment was complete surgery, with clear margins and lymphadenectomy. Similar to ocular melanomas, there were many debates about whether radical surgery is necessary or whether only a local excision is adequate. The conclusion of the majority of the studies published, as in ocular melanoma, is that a conservative approach has good results, and extensive surgery does not prolong survival. Unfortunately, because of its rarity, mucosal melanoma are never the first option in the list of clinicians' or pathologists' presumed diagnosis. It is very important to be aware that this entity exists, to biopsy all unusual tumors, and to avoid radical surgery. Then, together with the pathologist and oncologist, we can offer the patient the chance to benefit from the new schemes of therapy. ${ }^{58,59}$

In recent years, the discovery of genetic fingerprints in mucosal melanoma established new treatment strategies. The good response of gastrointestinal stromal tumors that harbor the mutations of kit gene to imatinib is already well-known. Kit gene mutations are responsible for tumor growth and progression. One-third of mucosal melanomas are c-kitpositive. Clinical trials on c-kit-positive mucosal melanomas showed a total disappearance of the tumor after 6 weeks of treatment. This response encourages physicians to classify mucosal melanomas on molecular grounds and to recommend this treatment to selected c-kit-positive patients. ${ }^{60,61}$

Even if a correct diagnosis is pronounced, the prognosis is grim, with 5 -year survival rates being around $40 \%$ compared with $90 \%$ for the mucosal melanomas' cutaneous counterparts. ${ }^{62}$

\section{Conclusion}

Mucosal melanomas are tumors associated with aging, and all our patients were older than 53 years. The poor prognosis of these tumors requires teamwork among the clinician, pathologist, radiologist, and oncologist to have an early and correct diagnosis. It is mandatory to establish the particularities of each case and offer the patient the best novel, personalized therapy.

\section{Disclosure}

The authors report no conflicts of interest in this work.

\section{References}

1. Fernandez-Flores A. Prognostic factors for melanoma progression and metastasis: from Hematoxylin-Eosin to genetics. Rom J Morphol Embryol. 2012;53(3):449-459.

2. Weber CO. Chirurgische Ehrfahrungen und Untersuchungen, nebst zahlreichen Beobachtungen aus der chirurgischen Klinik und dem Evan gelischen Krankenhaus zu Bonn. Berlin; G Reimer; 1859.

3. Lucke A. Die Lehre von den Geschwulsten in anatomischer und klinischer Beziehung in Handbuch d. allg. u. spec. chir., Erlangen, 1869;244.

4. Lincoln RP. A case of melano-sarcoma of the nose cured by galvanocauterization. $N$ Y Med J. 1885;42:406-407.

5. Curtin JA, Fridlyand J, Kageshita T, et al. Distinct sets of genetic alterations in melanoma. $N$ Engl J Med. 2005;353(20):2135-2147.

6. McLaughlin CC, Wu XC, Jemal A, Martin HJ, Roche LM, Chen VW. Incidence of noncutaneous melanomas in the US Cancer. 2005;103(5): $1000-1007$.

7. Mihajlovic M, Vlajkovic S, Jovanovic P, Stefanovic V. Primary mucosal melanomas: a comprehensive review. Int J Clin Exp Pathol. 2012;(8):739-753.

8. Chang AE, Karnell LH, Menck HR. The National Cancer Data Base report on cutaneous and noncutaneous melanoma: a summary of 84,836 cases from the past decade. The American College of Surgeons Commission on Cancer and the American Cancer Society. Cancer. 1998; 83(8):1664-1678.

9. Pandey M, Mathew A, Abraham EK, Ahamed IM, Nair KM. Primary malignant melanoma of the mucous membranes. Eur J Surg Oncol. 1998;24(4):303-307.

10. Takagi M, Ishikawa G, Mori W. Primary malignant melanoma of the oral cavity in Japan. With special reference to mucosal melanosis. Cancer. 1974;34(2):358-370.

11. Seetharamu N, Ott PA, Pavlick AC. Mucosal melanomas: a case-based review of the literature. Oncologist. 2010;15(7):772-781.

12. Broomhall C. Malignant melanoma of the oral cavity in Ugandan Africans. Br J Surg. 1967;54(7):581-584.

13. Axéll T, Hedin CA. Epidemiologic study of excessive oral melanin pigmentation with special reference to the influence of tobacco habits. Scand J Dent Res. 1982;90(6):434-442.

14. Liversedge RL. Oral malignant melanoma. Br J Oral Surg. 1975;13(1): $40-55$.

15. Manolidis S, Donald PJ. Malignant mucosal melanoma of the head and neck: review of the literature and report of 14 patients. Cancer. 1997;80(8):1373-1386.

16. Rapini RP, Golitz LE, Greer RO Jr, Krekorian EA, Poulson T. Primary malignant melanoma of the oral cavity. A review of 177 cases. Cancer. 1985;55(7):1543-1551.

17. Rapini RP. Oral melanoma: diagnosis and treatment. Semin Cutan Med Surg. 1997;16(4):320-322.

18. Chaudhry AP, Hampel A, Gorlin RJ. Primary malignant melanoma of the oral cavity: a review of 105 cases. Cancer. 1958;11(5):923-928.

19. Holmstrom M, Lund VJ. Malignant melanomas of the nasal cavity after occupational exposure to formaldehyde. Br J Ind Med. 1991;48(1): 9-11.

20. Thompson LD, Wieneke JA, Miettinen M. Sinonasal tract and nasopharyngeal melanomas: a clinicopathologic study of 115 cases with a proposed staging system. Am J Surg Pathol. 2003;27(5):594-611.

21. Davies H, Bignell GR, Cox C, et al. Mutations of the BRAF gene in human cancer. Nature. 2002;417(6892):949-954.

22. Cruz F 3rd, Rubin BP, Wilson D, et al. Absence of BRAF and NRAS mutations in uveal melanoma. Cancer Res. 2003;63(18):5761-5766.

23. Rimoldi D, Salvi S, Liénard D, et al. Lack of BRAF mutations in uveal melanoma. Cancer Res. 2003;63(18):5712-5715.

24. Debiec-Rychter M, Sciot R, Le Cesne A, et al; EORTC Soft Tissue and Bone Sarcoma Group; Italian Sarcoma Group; Australasian GastroIntestinal Trials Group. KIT mutations and dose selection for imatinib in patients with advanced gastrointestinal stromal tumours. Eur J Cancer. 2006;42(8):1093-1103. 
25. Curtin JA, Busam K, Pinkel D, Bastian BC. Somatic activation of KIT in distinct subtypes of melanoma. J Clin Oncol. 2006;24(26): 4340-4346.

26. Rivera RS, Nagatsuka H, Gunduz M, et al. c-kit protein expression correlated with activating mutations in KIT gene in oral mucosal melanoma. Virchows Arch. 2008;452(1):27-32.

27. Antonescu CR, Busam KJ, Francone TD, et al. L576P KIT mutation in anal melanomas correlates with KIT protein expression and is sensitive to specific kinase inhibition. Int J Cancer. 2007;121(2):257-264.

28. Furney SJ, Turajlic S, Stamp G, et al. Genome sequencing of mucosal melanomas reveals that they are driven by distinct mechanisms from cutaneous melanoma. J Pathol. 2013;230(3):261-269.

29. Van Raamsdonk CD, Bezrookove V, Green G, et al. Frequent somatic mutations of GNAQ in uveal melanoma and blue naevi. Nature. 2009; 457(7229):599-602.

30. Van Raamsdonk CD, Griewank KG, Crosby MB, et al. Mutations in GNA11 in uveal melanoma. N Engl J Med. 2010;363(23):2191-2199.

31. Patel SG, Prasad ML, Escrig M, et al. Primary mucosal malignant melanoma of the head and neck. Head Neck. 2002;24(3):247-257.

32. Balch CM, Gershenwald JE, Soong SJ, et al. Final version of 2009 AJCC melanoma staging and classification. J Clin Oncol. 2009;27(36): 6199-6206.

33. Hicks MJ, Flaitz CM. Oral mucosal melanoma: epidemiology and pathobiology. Oral Oncol. 2000;36(2):152-169.

34. Patrick RJ, Fenske NA, Messina JL. Primary mucosal melanoma. J Am Acad Dermatol. 2007;56(5):828-834.

35. Chalkiadakis G, Wihlm JM, Morand G, Weill-Bousson M, Witz JP Primary malignant melanoma of the esophagus. Ann Thorac Surg. 1985 39(5):472-475.

36. Christova S, Meinhard K, Mihailov I, Alexiev B. Three cases of primary malignant melanoma of the alimentary tract. Gen Diagn Pathol. 1996;142(1):63-67.

37. Kadivar TF, Vanek VW, Krishnan EU. Primary malignant melanoma of the small bowel: a case study. Am Surg. 1992;58(7):418-422.

38. Sachs DL, Lowe L, Chang AE, Carson E, Johnson TM. Do primary small intestinal melanomas exist? Report of a case. J Am Acad Dermatol. 1999;41(6):1042-1044.

39. Chandler AB, Jones GF. Malignant melanoma of the gastrointestinal tract; a case report. Am Surg. 1951;17(8):719-721.

40. Rao GM, Satyanarayana Y, Janaki M, Hayath MS. Primary melanocarcinoma of stomach. Indian J Gastroenterol. 1999;18(4):176.

41. Alazmi WM, Nehme OS, Regalado JJ, Rogers AI. Primary gastric melanoma presenting as a nonhealing ulcer. Gastrointest Endosc. 2003; 57(3):431-433.

42. Alazmi WM. Review. Gastroenterol Hepatol (N Y). 2008;4(11): 797-798.

43. Baderca F, Cojocaru S, Lazăr E, et al. Amelanotic vulvar melanoma: case report and review of the literature. Rom J Morphol Embryol. 2008; 49(2):219-228.

44. Ludgate MW, Fullen DR, Lee J, et al. Animal-type melanoma: a clinical and histopathological study of 22 cases from a single institution. $\mathrm{Br} J$ Dermatol. 2010;162(1):129-136.
45. Blecker D, Abraham S, Furth EE, Kochman ML. Melanoma in the gastrointestinal tract. Am J Gastroenterol. 1999;94(12):3427-3433.

46. Reintgen DS, Thompson W, Garbutt J, Seigler HF. Radiologic, endoscopic, and surgical considerations of melanoma metastatic to the gastrointestinal tract. Surgery. 1984;95(6):635-639.

47. Capizzi PJ, Donohue JH. Metastatic melanoma of the gastrointestinal tract: a review of the literature. Compr Ther. 1994;20(1):20-23.

48. Cheung MC, Perez EA, Molina MA, et al. Defining the role of surgery for primary gastrointestinal tract melanoma. J Gastrointest Surg. 2008;12(4):731-738.

49. Sugiyama VE, Chan JK, Shin JY, Berek JS, Osann K, Kapp DS. Vulvar melanoma: a multivariable analysis of 644 patients. Obstet Gynecol. 2007;110(2 Pt 1):296-301.

50. Ragnarsson-Olding BK, Kanter-Lewensohn LR, Lagerlöf B, Nilsson BR, Ringborg UK. Malignant melanoma of the vulva in a nationwide, 25-year study of 219 Swedish females: clinical observations and histopathologic features. Cancer. 1999;86(7):1273-1284.

51. Verschraegen CF, Benjapibal M, Supakarapongkul W, et al. Vulvar melanoma at the M. D. Anderson Cancer Center: 25 years later. Int $J$ Gynecol Cancer. 2001;11(5):359-364.

52. DiMarco DS, DiMarco CS, Zincke H, et al. Outcome of surgical treatment for primary malignant melanoma of the female urethra. $J$ Urol. 2004;171(2 Pt 1):765-767.

53. Gupta R, Bhatti SS, Dinda AK, Singh MK. Primary melanoma of the urethra: a rare neoplasm of the urinary tract. Int Urol Nephrol. 2007; 39(3):833-836.

54. Nakamoto T, Inoue Y, Ueki T, Niimi N, Iwasaki Y. Primary amelanotic malignant melanoma of the female urethra. Int $J$ Urol. 2007;14(2): 153-155.

55. Pacella M, Gallo F, Gastaldi C, Ambruosi C, Carmignani G. Primary malignant melanoma of the bladder. Int J Urol. 2006;13(5):635-637.

56. Baderca F, Mateş I, Solovan C. Unusual variant of blue nevus associated with dermatofibromas. Rom J Morphol Embryol. 2013;54(2): 413-417.

57. Baderca F, Solovan C, Boghian L. Epidemiological and morphological data of ocular melanocytic lesions. Rom J Morphol Embryol. 2013; 54(1):77-83

58. Phillips GL, Bundy BN, Okagaki T, Kucera PR, Stehman FB. Malignant melanoma of the vulva treated by radical hemivulvectomy. A prospective study of the Gynecologic Oncology Group. Cancer. 1994;73(10): 2626-2632.

59. Trimble EL. Melanomas of the vulva and vagina. Oncology (Williston Park). 1996;10(7):1017-1023.

60. Carvajal RD, Antonescu CR, Wolchok JD, et al. KIT as a therapeutic target in metastatic melanoma. JAMA. 2011;305(22):2327-2334.

61. Guo J, Si L, Kong Y, et al. Phase II, open-label, single-arm trial of imatinib mesylate in patients with metastatic melanoma harboring c-Kit mutation or amplification. J Clin Oncol. 2011;29(21):2904-2909.

62. Tomicic J, Wanebo HJ. Mucosal melanomas. Surg Clin North Am. 2003;83(2):237-252.
Clinical Interventions in Aging

\section{Publish your work in this journal}

Clinical Interventions in Aging is an international, peer-reviewed journal focusing on evidence-based reports on the value or lack thereof of treatments intended to prevent or delay the onset of maladaptive correlates of aging in human beings. This journal is indexed on PubMed Central, MedLine,

\section{Dovepress}

CAS, Scopus and the Elsevier Bibliographic databases. The manuscript management system is completely online and includes a very quick and fair peer-review system, which is all easy to use. Visit http://www.dovepress. com/testimonials.php to read real quotes from published authors. 